\title{
GEOMETRIC RELATIONS BETWEEN HOMEOMORPHIC RIEMANN SURFACES
}

\author{
BY A. MARDEN ${ }^{1}$
}

In complex analysis one often investigates a particular class of functions on some domain. More often than not the domain is simply connected and if it is not the whole plane, it is usually taken to be the unit disk. For this is conformally equivalent to any other nondegenerate simply connected region and the conformal map induces a certain isomorphism of the given class of functions to a class on the disk. What doesn't go over nicely, in general, are boundary properties of functions.

On the other hand if the domain is multiply connected, it is no longer true that any two nondegenerate ones of the same connectivity are conformally equivalent. Thus before we can start to examine deeper relations between corresponding classes of functions, we must understand how the domains themselves are related.

Teichmüller space is a space of domains all of the same topological type. In that theory we find out how the domains are related to each other and then parametrize them. At least this is true for domains of finite connectivity. Once we do this, we can understand, for example, how the canonical domain functions like the harmonic measures vary real analytically in the parameters.

The foundation of Teichmüller space theory is thus rooted in methods for comparing two domains of the same topological type. The theory is most completely worked out and in any case has the nicest expression when the domains involved have no boundary at all; that is are compact Riemann surfaces without boundary. For this reason our explanations will ultimately be restricted to this case.

We start with two Riemann surfaces $R$ and $S$ and an (orientation preserving) homeomorphism $f: R \rightarrow S$. Since we are really going to deal with conformal equivalence classes, if $f$ is homotopic to a conformal map we consider that $S$ is the same as $R$ and $f$ is the identity. Of course given two surfaces $R, S$, there are in general infinitely many choices for homeomorphisms $f$, no two being homotopic. For example, here are two; the image regions are the same in both cases but the two images of the arc between punctures lie in different homotopy classes.

An invited hour address presented to the American Mathematical Society at Kent, Ohio, November 3, 1979; received by the editors April 15, 1980.

1980 Mathematics Subject Classification. Primary 30F99; Secondary 30-02, 30C60, 30F35.

'Supported in part by the National Science Foundation. 

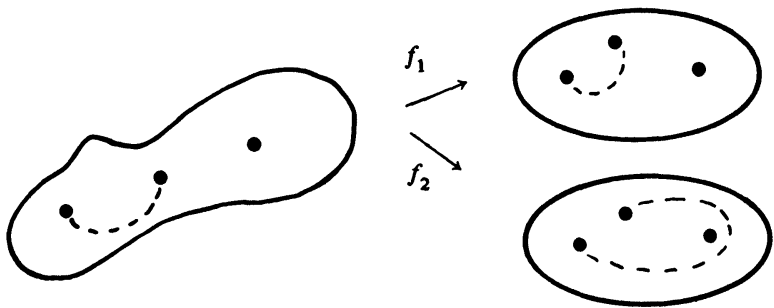

The number of choices of $f$ corresponds to the number of essentially different ways that $S$ can be cut so as to become simply connected.

We will present two methods for relating $S$ to $R$ from the point of view of $f$ and briefly describe some recent advances which clarify each of them.

I. $S$ arises from $R$ by a generalized affine stretch homotopic to $f$. The parameters in the stretch describe the relative geometry. This method originates with Teichmüller.

II. By means of $f, R$ and $S$ become parallel boundary components of a uniquely determined hyperbolic 3-manifold. This is in analogy with the picture of two parallel closed surfaces in euclidean space, bounding the region between them, $f$ being the map along normals, sending one surface to the other. The geometry of the hyperbolic 3-manifold then describes the relative geometry of $R$ and $S$. This method originates with Bers.

Now we will describe these methods in some detail.

METHOD I. Here everything is based on the simplest possible nonconformal map, the affine map. We write it in normalized complex form,

$$
A(z)=\frac{z+k e^{i \theta} \bar{z}}{\sqrt{1-k^{2}}}, \quad 0<k<1,
$$

where the condition on $k$ is determined by the requirement that $A(z)$ be orientation preserving and nonsingular. Geometrically, $A$ is a stretch of magnitude $\sqrt{K}=[(1+k) /(1-k)]^{1 / 2} \geqslant 1$ along the parallel family of lines inclined at angle $\theta / 2$ to the real axis, and compression of magnitude $1 / \sqrt{K}$ along the perpendicular lines. Thus,
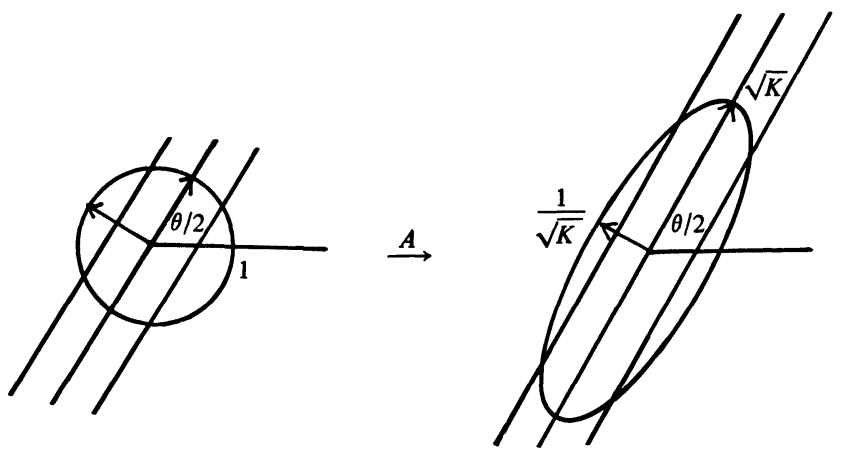

The next step is to explain what is meant by a generalized affine stretch. Suppose the affine stretch $A$ has been applied to a particular plane region $R_{1}$ yielding the image region $S_{1}$. Suppose we take conformal maps $\Phi: R \rightarrow R_{1}$ 
and $\Psi: S_{1} \rightarrow S$ and then consider the composition

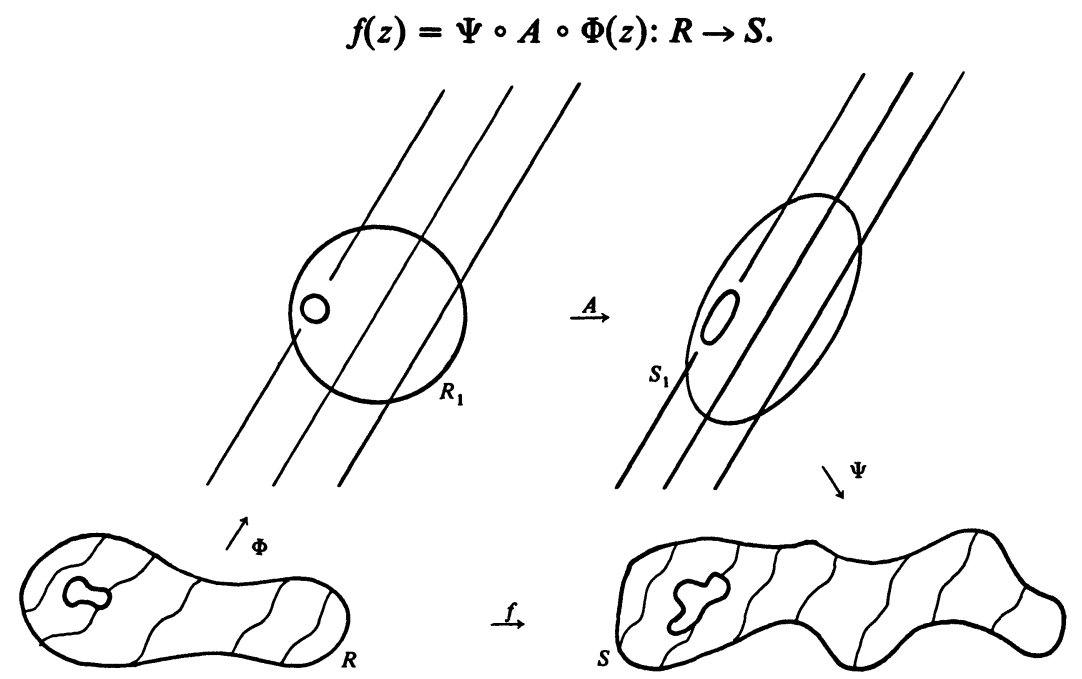

With respect to its nonconformal character, we can describe $f$ as an affine stretch of magnitude $\sqrt{K}$ along the lines

$$
\left\{z \in R: \operatorname{Im} e^{i-\theta / 2} \Phi(z)=\text { const }\right\},
$$

the pull-back under $\Phi$ of the stretch lines for $A$.

Such maps $f$ are examples of Teichmüller maps. The manner in which we have introduced them here is however too restrictive. They are defined so that locally at nonsingular points they have the structure described, not necessarily globally.

The general definition is formulated as follows. The map $f$ we have just constructed solves the Beltrami equation on $R$,

$$
f_{\bar{z}}=\mu f_{z}, \quad \mu(z)=k e^{i \theta} \frac{\overline{\left(\Phi^{\prime}\right)^{2}}}{\left|\left(\Phi^{\prime}\right)^{2}\right|}(z)=k \frac{\overline{q(z)}}{|q(z)|},
$$

where $q(z)=Q^{\prime}(z)^{2}, Q(z)=e^{-i \theta / 2} \Phi(z)$. From a more general point of view we learn to think of the analytic function $q(z)$ as a quadratic differential on $R$ with invariant expression $q(z) d z^{2}$ under local coordinate changes or global conformal maps. The stretch lines for $f$ can be described in terms of $q$ as

$$
\left\{z \in R: q(z) d z^{2}>0\right\}=\{z \in R: \operatorname{Im} Q(z)=\text { const }\} .
$$

These are called trajectories (or more precisely, horizontal trajectories) of the differential $q$. For the general theory of trajectories we refer to [20], [21].

Now turn around and, given an analytic function $q(z)$ on $R$-or quadratic differential $q d z^{2}$ if $R$ is not a planar Riemann surface, consider the Beltrami equation on $R$,

$$
f_{\bar{z}}=\mu f_{z}, \quad \mu=k \overline{q(z)} /|q(z)|, \quad 0<k<1
$$

(the condition that $q$ is a quadratic differential makes this invariantly defined on the Riemann surface $R$ ). It has a solution $f$ which is a homeomorphism of 
$\boldsymbol{R}$ onto a Riemann surface $f(R)$ and is uniquely determined up to composition on the left with a conformal map. If $U \subset R$ is a small neighborhood without zeros of $q(z)$ then in $U, f$ has the form

$$
\begin{gathered}
f(z)=\Psi \circ A \circ Q(z), \\
Q(z)=\int^{z} \sqrt{q} d z, \quad A(z)=(z+k \bar{z}) / \sqrt{1-k^{2}}
\end{gathered}
$$

and $\Psi$ is conformal on $A \circ Q(U) ; \Psi$ plays the less essential role of a normalizing term. Globally, the solution $f$ is obtained by patching together these little pieces.

At a zero of order $p, q(z) \sim z^{p}$ and $f \sim\left[A\left(z^{(p+2) / 2}\right)\right]^{2 /(p+2)}$ is a local homeomorphism. The general theory also allows for simple poles $p=-1$ since the area $\iint|q| d x d y$ is still locally finite. There are $(p+2)$ horizontal trajectories-essentially the rays $\left\{z \in C: \operatorname{Im} z^{(p+2) / 2}=0\right\}$ - emanating from the zero (or simple pole). These are called critical trajectories.

With this said, we think of $f$ as an affine stretch of magnitude $\sqrt{K}$, $K=(k+1) /(k-1)$ along the trajectories $\left\{z: q(z) d z^{2} \geqslant 0\right\}$ of $q$. Moreover the image Riemann surface $f(R)$ can be constructed from $q(z)$ and $k$ by patching together local coordinate neighborhoods $\{A \circ Q(U)\}$, making due allowance for the singular points. This process will be illustrated below.

By introducing a parameter $t$ in $t k, 0<t<1$, we can interpolate cóntinuously between $R$ and $f(R)$.

For an example consider the situation presented in this diagram

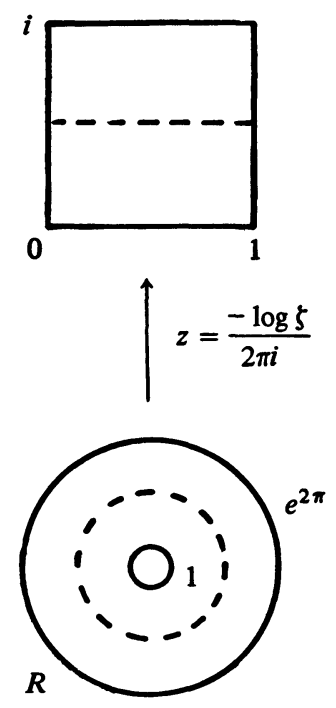

$$
w=\frac{z+k \bar{z}}{\sqrt{1-k^{2}}}
$$
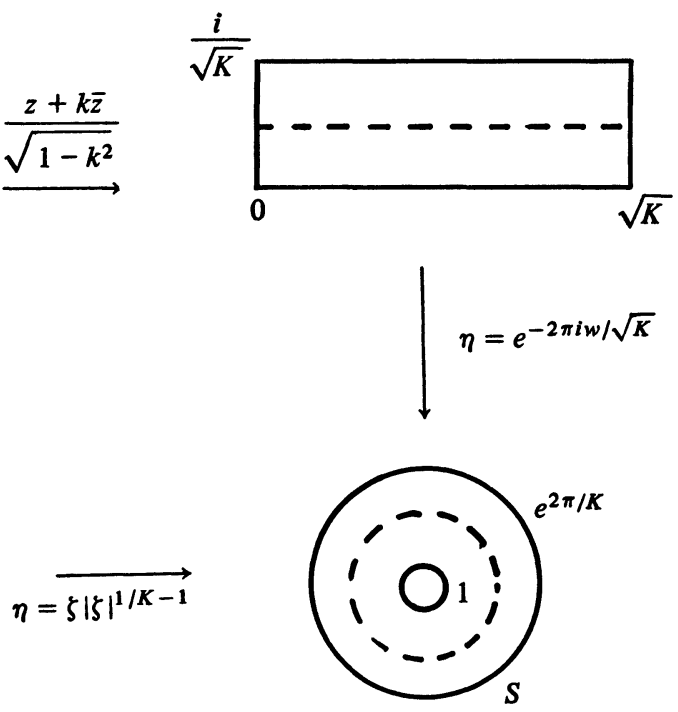

$\eta$ satisfies the Beltrami equation on $\boldsymbol{R}$ with

$$
\mu(\zeta)=k \frac{\bar{q}(\zeta)}{|q(\zeta)|}, \quad q(\zeta)=-\frac{1}{\zeta^{2}}
$$

On the circles $\zeta=r e^{i \theta}, q(\zeta) d \zeta^{2}=d \theta^{2}>0$. In this manner the affine stretch is realized in the annulus as a stretch along the concentric circles. 
Now return to a general surface or plane region $R$. The construction we have described corresponding to a chosen differential $q$ is beautifully explicit in the following circumstances.

Suppose $R$ is any Riemann surface and $\gamma$ is a nondegenerate simple closed curve on $R$, that is, not retractable to a point or a puncture. Associated with $\gamma$ is the Jenkins-Strebel quadratic differential $\varphi[\gamma] d z^{2}$ (see [10], [20]). This remarkable differential is uniquely determined up to positive scalar multiple by the following properties. Cut $R$ along all of the critical trajectories (globally extended) of $\varphi[\gamma]$. Then what is left is an annular region whose central curves are freely homotopic to $\gamma$. Consequently

$$
\Phi(z)=b \exp c \int^{z} \sqrt{\varphi[\gamma]} d z \text { for suitable constants } b, c
$$

maps the cut $R$ conformally onto an annulus. For instance,
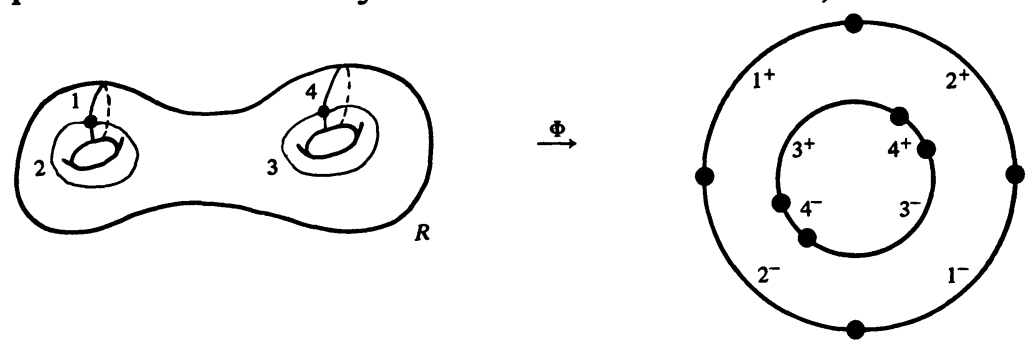

In this mapping, opposite sides of an arc running along a critical trajectory from one critical point to another are sent to two boundary arcs of the annulus which subtend the same angles at the center.

Occasionally it is possible to write down the $J-S$ differential explicitly. For example, for the 4-punctured sphere and $\gamma$ as shown,

$$
\varphi[\gamma] d z^{2}=\frac{d z^{2}}{z(z-1)(z-2)}
$$

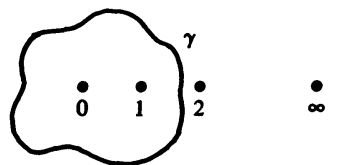

and the critical trajectories run along $\mathbf{R}$ from 0 to 1 and 2 to $\infty$. For the torus generated by a square lattice and $\gamma$ the simple loop arising from the line segment to $m+n i, m, n$ relatively prime, in terms of its representation in the plane,

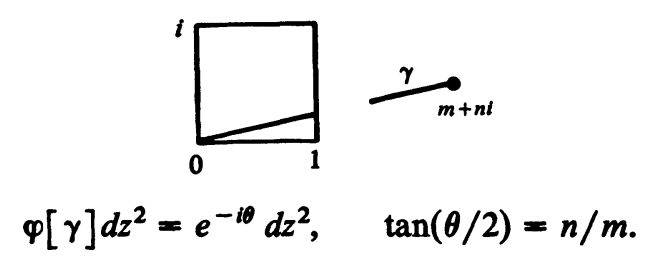

Here there are no critical trajectories (but only because the genus is one) so to obtain our annulus we must remove one of the mutually parallel closed trajectories in the free homotopy class determined by $\gamma$. 
Returning to our general surface $\boldsymbol{R}$ cut along the critical trajectories of $\varphi[\gamma]$, the modulus of the image annulus under $\Phi$, that is, $M(\gamma)=(1 / 2 \pi) \log r$ where $r$ is the outer radius of the annulus (the inner is 1 ) is called the modulus of the free homotopy class determined by $\gamma$. It is of course a conformal invariant. It can also be described [10] as the reciprocal of the extremal length of the family of curves freely homotopic to $\gamma$.

Now consider the Beltrami equation on $R$,

$$
f_{\bar{z}}=k \frac{\overline{\varphi[\gamma]}}{|\varphi[\gamma]|} f_{z}, \quad 0<k<1 .
$$

We can explicitly describe the corresponding map $f$ and the image surface $f(R)$. Namely we can express $f$ as the composition, $f=\Psi \circ \mathbb{Q} \circ \Phi$ where $\Phi$ is the map of the cut $R$ onto the annulus, $\mathbb{Q}$ is the radial contraction of it,

$$
\mathcal{Q}(\zeta)=\zeta|\zeta|^{1 / K-1}, \quad K=K_{f}=(1+k) /(1-k),
$$

and $\Psi$ is the conformal map of the image annulus into that Riemann surface obtained by identifying the opposite arcs on its boundary. Thus expressed in the form of a diagram,
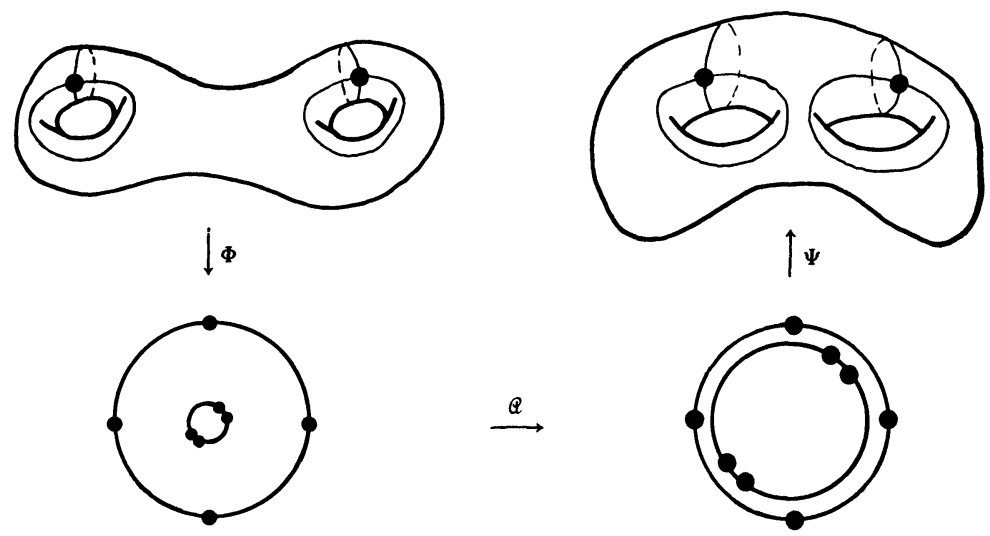

Now we return to our original problem. We are given two compact Riemann surfaces without boundary and (we can assume) a quasiconformal map $f$ between them:

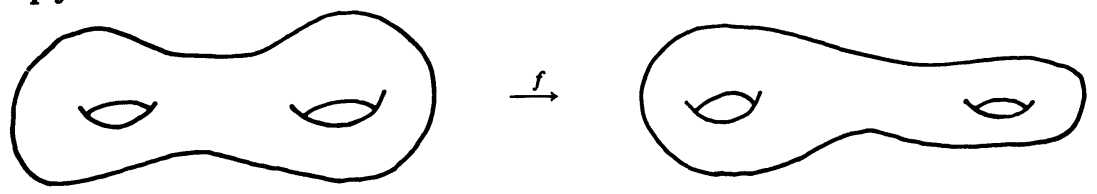

According to a celebrated theorem of Teichmüller (see [4]), $f$ is homotopic to, hence we may assume equal to the solution of a Beltrami equation,

$$
f_{\bar{z}}=k \frac{\bar{\varphi}}{|\varphi|} f_{z}, \quad K_{f}=(1+k) /(1-k), \quad 0<k<1
$$

for some quadratic differential $\varphi$ on $R$. Furthermore, $\varphi$ is uniquely determined up to a positive scalar factor by the homotopy class of $f$. This means $f$ is a generalized affine stretch of magnitude $\sqrt{K_{f}}$ along the trajectories of $\varphi$. 
Teichmüller's theorem would remain true if $R$ were a finitely punctured closed surface-like the $n$-punctured sphere-but then $\varphi$ may have simple poles at the punctures.

Now we ask, how is $\varphi$ determined geometrically by the initial surface $R$ and the homotopy class of $f$ ?

In the simplest case, $f$ is an annular map associated with $\varphi=\varphi[\gamma]$ for some $\gamma$ on $R$. The image surface $S$ can be explicitly constructed from $R$, given $k$, as before. In general, however, the situation is as follows.

Define a number $K$ in terms of the extremal problem

$$
1 / K=\inf _{\delta} M(f(\delta))_{S} / M(\delta)_{R} \quad\left(>1 / K_{f}\right)
$$

where the infimum is taken over all simple nondegenerate curves $\delta$ on $R$. The inequality is known to be true generally and the ratio $M(f(\delta)) / M(\delta)=K_{f}^{-1}$ if and only if $\varphi=\varphi[\gamma]$ for some $\gamma$ and $\delta=\gamma$.

Choose any sequence of simple curves $\left\{\gamma_{n}\right\}$ on $R$ with $\lim M\left(f\left(\gamma_{n}\right)\right) / M\left(\gamma_{n}\right)$ $=K^{-1}$. Normalize the differentials $\varphi$ and $\left\{\varphi\left\{\gamma_{n}\right\}\right\}$ to have unit area $\iint_{R}|\cdot| d x d y=1$. The following statement involves results of Thurston [22], Kerckhoff [12], Masur [18], and the author's joint work with Strebel [15].

FACT. $\operatorname{Lim} \varphi\left[\gamma_{n}\right]=\varphi, K=K_{f}$, and the minimizing sequence $\left\{\gamma_{n}\right\}$ is uniquely determined "asymptotically".

We can picture this as follows. Consider the map $f_{n}$ constructed as the solution of

$$
w_{\bar{z}}=k \frac{\overline{\varphi\left[\gamma_{n}\right]}}{\left|\varphi\left[\gamma_{n}\right]\right|} w_{z}, \quad k=(K-1) /(K+1) .
$$

Then $f_{n}: R \rightarrow R_{n}$ is an affine stretch of magnitude $\sqrt{K}$ along the trajectories of $\varphi\left[\gamma_{n}\right]$ resulting in the surface $R_{n}$. The complement of the critical trajectories of $\varphi\left[\gamma_{n}\right]$ in $R$ is an annular region which is swept out by closed trajectories of $\varphi\left[\gamma_{n}\right]$ which are in the free homotopy class of $\gamma_{n}$. As $n$ increases, in general, the annular region becomes longer and thinner, while all the time filling the surface $R$. The simple closed curves sweeping out the annular regions converge to the noncritical trajectories of the limit differential $\varphi$. In general, however, these are not closed curves but rather determine a "measured foliation" of $R$ in the sense of Thurston. Meanwhile, the image surfaces $R_{n}$ converge to $S$. We summarize in the following diagram:

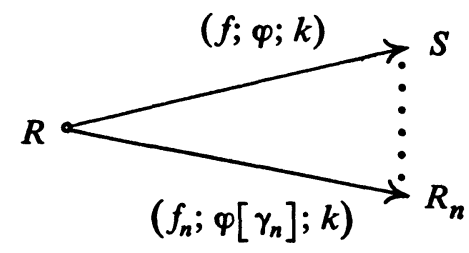

Using an idea of Thurston, the sequence $\left\{\gamma_{n}\right\}$ can be characterized geometrically as follows.

$$
\inf _{\alpha_{0} \sim \alpha} \int_{\alpha_{0}}|\operatorname{Im} \sqrt{\varphi} d z|=\lim _{n \rightarrow \infty} \frac{i\left(\alpha, \gamma_{n}\right)}{a_{n}}, \text { for all } \alpha \text { on } R
$$


where the integral is the total variation of $|\operatorname{Im} \sqrt{\varphi} d z|=|\operatorname{Im} d Q|$ over $\alpha_{0}$ as $\alpha_{0}$ runs through the free homotopy class of $\alpha$ and $i\left(\alpha, \gamma_{n}\right)$ denotes the geometric intersection number. This is the smallest possible number of times curves freely homotopic to $\alpha$ and to $\gamma_{n}$ cross each other, or alternatively, the number of times the Poincare geodesic in the free homotopy class of $\alpha$ crosses that in the free homotopy class of $\gamma_{n}$. Finally $\left\{a_{n}\right\}$ is a sequence of positive numbers essentially depending only on $\left\{\gamma_{n}\right\}$. That is, if $\left\{b_{n}\right\}$ is any positive sequence such that

$$
\lim i\left(\alpha, \gamma_{n}\right) / b_{n}
$$

exists for all $\alpha$ and is $\neq 0$ for some $\alpha$ then

$$
\lim a_{n} / b_{n}=c>0
$$

exists.

In other words, $\varphi$ determines, and in fact is completely determined by, the sequence $\left\{\gamma_{n}\right\}$ of simple closed curves on $R$. The sequence $\left\{\gamma_{n}\right\}$ is in turn determined by $R, S$ and the homotopy class of $f$. The Teichmüller map $f$ is the generalized affine stretch of magnitude $\sqrt{K}$ in the "direction" $\lim \gamma_{n}$.

If $f$ actually maps $R$ onto itself then we can also consider the one-to-one correspondence $f_{*}: \varphi[\gamma] \rightarrow \varphi[f(\gamma)]$ between the totality of Jenkins-Strebel differentials on $R$. Because the differentials $\{\varphi[\gamma]\}$ are dense [18], using the technique suggested previously, $f_{*}$ extends to a homeomorphism $f_{*}$ of the real $(6 g-7)$-dimensional sphere $\Sigma$ of all normalized quadratic differentials on $R$ to itself. Suppose that no iterate of $f$ is homotopic to the identity and that $f$ does not preserve any collection of mutually disjoint simple loops. Then according to Thurston [22], $f_{*}$ has exactly two fixed points on $\Sigma$. In general, however, these differ from the Teichmüller differential associated with $f$.

This completes my description of how one surface is obtained from another by a generalized affine stretch along the trajectories of a quadratic differential. The point of view I have expressed, of basing the discussion on JenkinsStrebel differentials, originates in Masur's thesis [17] where the explicit nature of their trajectory structure was used to obtain the first precise information concerning the asymptotic behavior of at least some geodesic rays in Teichmüller space. More recent work of Hubbard and Masur [9] (see also [12], [22]) shows how any quadratic differential on $R$ can be completely characterized by the metrical/topological properties of its trajectory structure. Above all, Thurston's studies [22] of measured foliations have influenced all recent work in this area.

METHOD II. I want to start by explaining what I mean by two surfaces being parallel. In euclidean space if we take a closed, compact surface $R$ and move out a short distance along the normals we get a parallel surface $S$. The space in between is homeomorphic to $R \times[0,1]$. The natural map $f: R \rightarrow S$ obtained by moving along normals has the property that for any simple curve $\gamma \subset R, \gamma$ and $f(\gamma) \subset S$ are the boundary components of a cylinder in the 3-manifold.

Returning to our set-up $f: R \rightarrow S, R$ a closed, compact surface, we will find a natural 3-manifold $\mathscr{N}$ such that (i) $\partial \mathscr{N}=R \cup S$, (ii) $\mathscr{N} \approx R \times[0,1]$, and (iii) $\gamma \subset R$ and $f(\gamma) \subset S$ bound a cylinder in $\Re$ for any simple curve $\gamma$ 
on $R$, by moving along the fibers $y \times[0,1], y \in \gamma$. Strictly speaking, to carry this out we have to reverse the orientation of $f$.

To understand how this representation is obtained, start by realizing $R$ in its universal covering surface, which we take to be the upper half-plane $\mathbf{H}_{+}$, under a fuchsian group $\Gamma, \boldsymbol{R}=\mathbf{H}_{+} / \Gamma . \Gamma$ also acts in the lower half-plane $\mathbf{H}_{-}$ and this action is symmetric under reflection. The corresponding map $J$ : $\boldsymbol{R}^{\prime}=\mathbf{H}_{-} / \Gamma \rightarrow \boldsymbol{R}$ is anticonformal.
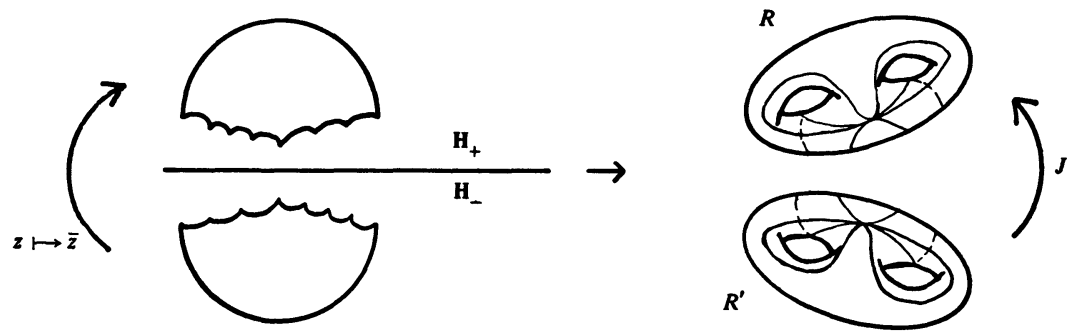

Now each Möbius transformation $z \rightarrow(a z+b) /(c z+d), a d-b c \neq 0$ is the restriction to the complex plane of a uniquely determined Möbius transformation that maps the upper half 3-space $\mathbf{H}^{3}$ onto itself. There is an elegant formula for it if one uses quaternions $(\vec{i}, \vec{j}, \vec{k})$ and for $z=x+i y \in \mathbf{C}$ writes the point over it $(z, t) \equiv(x, y, t) \in \mathbf{H}^{3}$ as

$$
\vec{h}=z+t \vec{j}=x+y \vec{i}+t \vec{j}+0 \vec{k} \text {. }
$$

Then the Möbius transformation in $\mathbf{H}^{3}$ is

$$
\vec{h} \rightarrow \overrightarrow{h^{\prime}}=(a \vec{h}+b)(c \vec{h}+d)^{-1} \text {. }
$$

The extension is conformal and an isometry in the hyperbolic metric $d s / t$ which is complete in $\mathbf{H}^{3}$.

In any case $\Gamma$ can be extended to $\mathbf{H}^{3}$ and since it then consists of conformal transformations preserving $R, \Gamma$ preserves each half-plane in $\mathbf{H}^{3}$ inclined to the real axis $\mathbf{R}$.

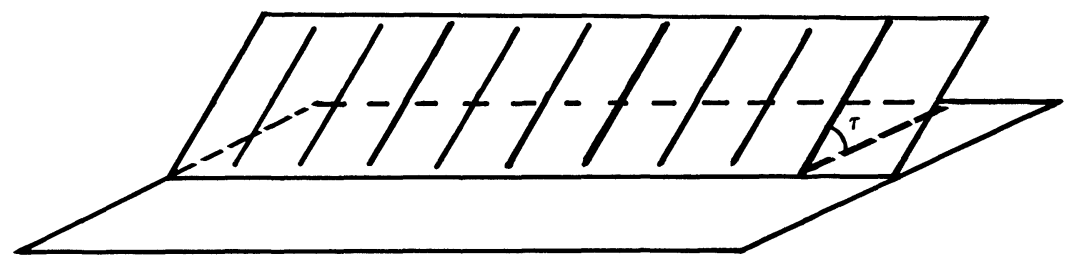

The quotient is a 3-manifold $\mathfrak{T}(\Gamma)$,

$$
\mathscr{R}(\Gamma)=\mathbf{H}^{3} \cup \mathbf{H}_{+} \cup \mathbf{H}_{-} / \Gamma \simeq R \times[0,1]
$$

with $\tau / \pi \in[0,1]$ parametrizing the parallel sheets which fill up $\mathscr{T}(\Gamma)$. Each of these is the quotient with $\Gamma$ of a half-plane inclined at some angle $\tau$ to $\mathbf{R}$. The interior of $\Re(\Gamma)$ is a complete hyperbolic manifold while its boundary is the union of the two Riemann surfaces $R, R^{\prime}$ whose conformal structure comes from $\mathbf{H}_{+} \cup \mathbf{H}_{-}$. The reflection $z \rightarrow \bar{z}$ extends to $\mathbf{H}^{3}$ as the reflection about a vertical plane and therefore the map $J: R^{\prime} \rightarrow R$ extends to an involution of $\mathscr{T}(\Gamma)$ pointwise fixing the middle sheet $R \times\{1 / 2\}$. For each 
simple closed curve $\gamma \subset R^{\prime}$ the cylinder $\{J(y) \times[0,1]: y \in \gamma\}$ in $\mathscr{N}$ is bounded by $\gamma$ and $J(\gamma)$.

Choose a standard set of generators for $\Gamma$,

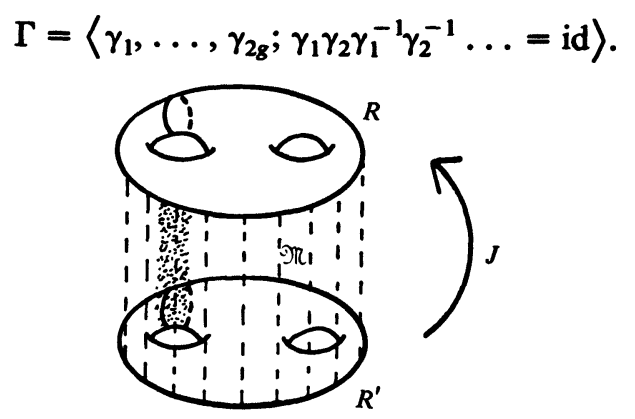

Vary the coefficients of the $\gamma_{j}$ not only in $\mathbf{R}$ but also in $\mathbf{C}$, keeping the relation satisfied. As we do this on the one hand we sweep out a connected open set in this coefficient space, and on the other hand the corresponding groups fill up what is called the quasifuchsian space of kleinian groups. First we will describe the situation locally, then globally.

As the coefficients of the generators $\left\{\gamma_{j}\right\}$ vary slightly but still satisfy the relation, the new transformations generate a new kleinian group which is the isomorphic image $\theta(\Gamma)$ of $\Gamma$. In fact, the isomorphism $\theta$ is geometrically determined in the following manner. There is a quasiconformal map $G$ of $\mathbf{H}^{3} \cup \mathbf{C} \cup\{\infty\}$ onto itself which satisfies

$$
(G \circ \gamma)(x)=\theta(\gamma) \circ G(x) \text { for all } x \in \mathbf{H}^{3} \cup \mathbf{C} \cup\{\infty\} .
$$

This means that instead of preserving $R \cup\{\infty\}, \theta(\Gamma)$ preserves the Jordan curve $G(R \cup\{\infty\})$, and instead of preserving a half-plane inclined to $R, \theta(\Gamma)$ preserves a crinkled half-plane inclined to $G(R \cup\{\infty\})$ :

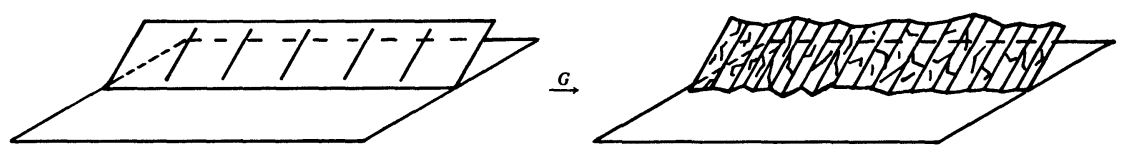

Furthermore the map $G$ projects to a homeomorphism

$$
g: \Re(\Gamma) \rightarrow \Re(\theta)=G\left(\mathbf{H}_{+}\right) \cup G\left(\mathbf{H}_{-}\right) \cup \mathbf{H}^{3} / \theta(\Gamma),
$$
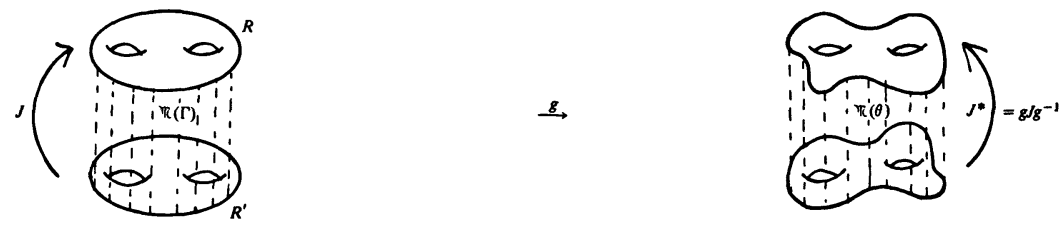

The two Riemann surfaces $G\left(\mathrm{H}_{+}\right) / \theta(\Gamma)$ and $G\left(\mathrm{H}_{-}\right) / \theta(\Gamma)$ are tied together by the fact they bound a (complete) hyperbolic 3-manifold which is symmetric under $J^{*}$.

Being quasiconformal deformations of the fuchsian group $\Gamma$, the groups $\theta(\Gamma)$ are called quasifuchsian. The set of all such groups-that is quasifuchsian space-can be characterized as follows. 
Let $\left(S_{0}, S, J^{*}\right)$ be any triple consisting of two Riemann surfaces $S_{0}, S$ of genus $g \geqslant 2$ and $J^{*}: S_{0} \rightarrow S$ an orientation reversing homeomorphism. There exists a quasifuchsian group $\theta(\Gamma)$, uniquely determined up to conjugation, such that $\mathscr{N}(\theta)$ represents the triple in the manner just described:
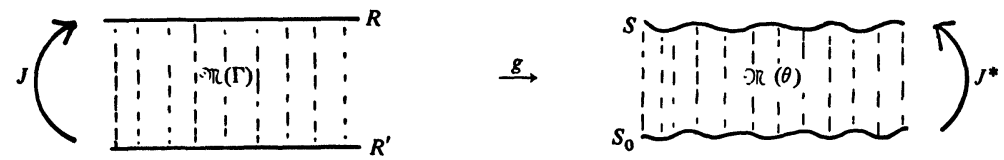

More formally, there are conformal maps $h_{0}, h$ of the components of $\partial \mathscr{T}(\theta)$ onto $S_{0}$ and $S$ respectively such that

$$
h g J g^{-1} h_{0}^{-1} \sim J^{*} .
$$

This process is called simultaneous uniformization because the two surfaces $S_{0}, S$ are being uniformized by a single group.

We have just introduced the quasifuchsian space of kleinian groups. For any kleinian group, in particular for the quasifuchsian groups $\theta(\Gamma)$, a model for Int $\mathscr{R}(\theta)$ can be constructed in $\mathbf{H}^{3}$ by taking the Poincare fundamental polyhedron $\mathscr{P}(\theta)$ with some prescribed center, say $O$. Its faces are automatically arranged in pairs and when these are identified, Int $\Re(\theta)$ results. The intersection of the euclidean closure $\mathscr{P}(\theta)^{-}$of $\mathscr{P}(\theta)$ with $\mathbf{C} \cup\{\infty\}$ is a union of polygonal faces. The sides of these are arranged in pairs, and when these are identified, $\partial \mathscr{T}(\theta)$ results. But also from the point of view of the fundamental polyhedron we can define what it means for a sequence of hyperbolic 3-manifolds $\left\{\mathfrak{T}_{n}\right\}$ to converge to another one $\mathfrak{T}$ : Namely the corresponding sequence of fundamental polyhedra representing $\mathfrak{N}_{n}$ converges to that representing $\Re$. This phenomenon is called geometric convergence of groups and was first described explicitly by Jorgensen [11]. We omit mention of many details.

The basic theory of quasifuchsian groups originates from work of Bers [3] and Maskit [16] and the study of these groups in space relies on Gehring's extension theorem [8]. The original formulation of simultaneous uniformization by Bers was entirely in terms of the two bounding surfaces $\partial \mathscr{T}(\theta)$. However in joint work with Earle [7] we find a great deal of additional structure by focusing on the entire manifold $\Re(\theta)$. We will see an example later.

In any case, it is time to return to the original question of describing the relationship $f: R \rightarrow S$. Apply simultaneous uniformization to the triple,

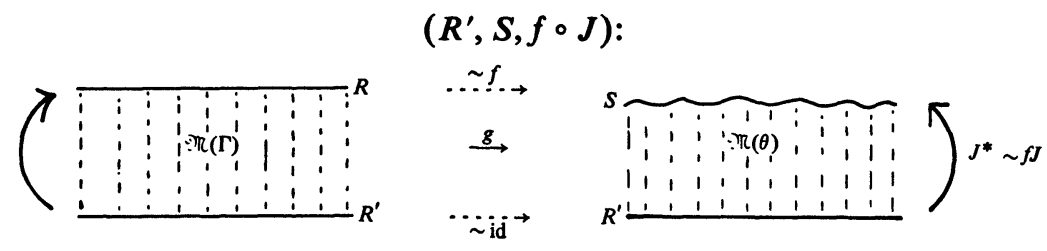

The diagram is meant to suggest that the restrictions of $g$ to $R^{\prime}$ and $R$ are homotopic to the maps identity and $f$ respectively. The geometry of the relation $f: R \rightarrow S$ is now contained in the geometry of the quasifuchsian group $\theta(\Gamma)$ or hyperbolic 3-manifold $\Re(\theta)$. 
This description is perhaps not as immediately appealing as that of Method I. On the other hand, unlike Method I, it is intimately and directly connected with the analytic structure of Teichmüller space. The complex parameters in the generators $\left\{\theta\left(\gamma_{j}\right)\right\}$ not only give rise to a set of analytic parameters but their natural domain of existence is relatively compact. Its boundary is therefore a very natural boundary of Teichmüller space: it is called the Bers boundary.

Application. Now we will see these two methods in action together. There are two basic operations on plane domains or Riemann surfaces: pinching and twisting. We will compare how each is handled by the methods.

In describing the two operations, for simplicity and definiteness, we will deal with a surface $R$ of genus two, and a simple curve $\gamma$ cutting $R$ into two halves.

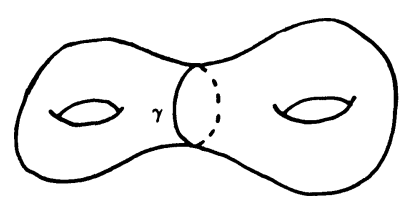

Pinching. Cut out a thin annular neighborhood about $\gamma$ and sew in ever thicker ones in its place (larger modulus). We get a one (real) parameter family $f_{t}: R \rightarrow R_{t}$ depending on the choice of annular neighborhood that looks as follows:

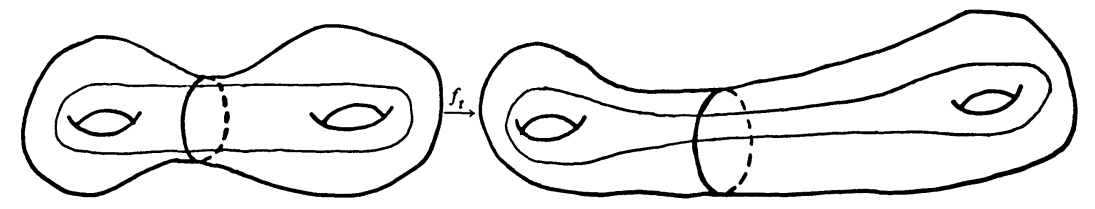

Twisting. A twist of an annulus is the following homeomorphism of an annulus onto itself that leaves its boundary pointwise fixed. The inner contour is kept fixed while the outer is rotated counterclockwise by $360^{\circ}$. An arc between the boundary contours

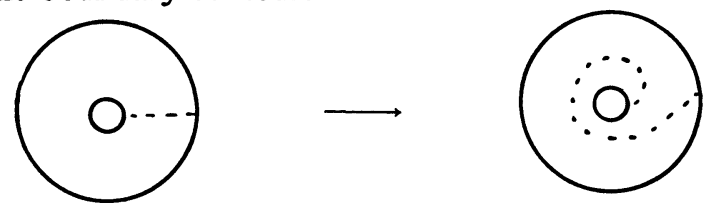

becomes twisted around once. Now on the surface $R$, take a thin annular region about $\gamma$, apply the twist in this, and extend it to all $R$ by setting it equal to the identity outside. There results a homeomorphism $T: R \rightarrow R$ called a Dehn twist which is not homotopic to the identity and whose homotopy class depends only on the free homotopy class of $\boldsymbol{\gamma}$.
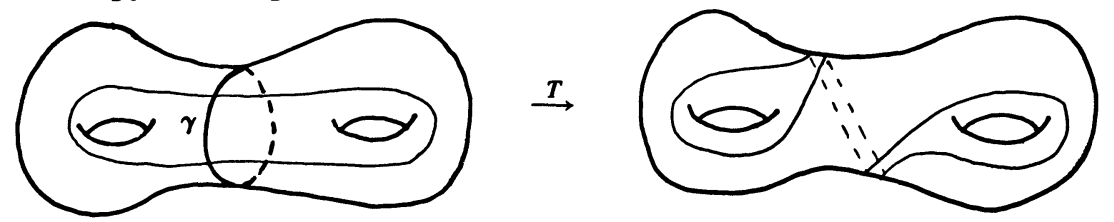

Of course $T$ can be iterated. 
MeTHOD I. We will construct from $R$ and $\gamma$ a canonical one-parameter family of pinched surfaces $\left\{\boldsymbol{R}_{t}\right\}$. For this family and then also for the Dehn twist, we will explicitly display the generalized affine stretches (Teichmüller maps) in the homotopy classes. Let $\varphi[\gamma]$ denote the Jenkins-Strebel differential corresponding to $\gamma$. Consider the Beltrami equation,

$$
f_{\bar{z}} / f_{z}=-t \overline{\varphi[\gamma]} /|\varphi[\gamma]|, \quad 0<t<1 .
$$

We get the following picture, letting $f_{t}$ denote a solution and $t \rightarrow 1$.
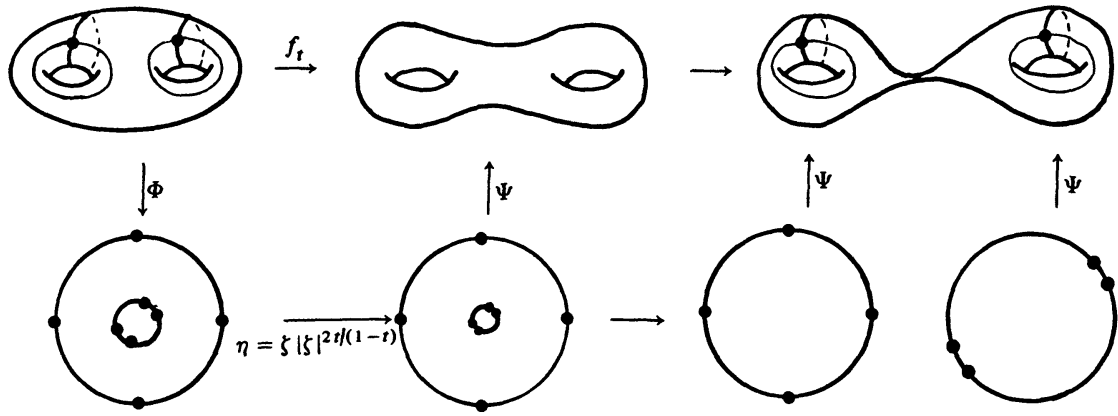

In terms of the annular image of the cut $R$, we have the radial stretch. At the last step when the annulus becomes ever thicker the outer contour becomes the point at $\infty$. However had we normalized the annulus so that the outer contour is of unit radius, then the inner contour approaches the origin. Consequently we say that the limit results in two once-punctured disks, one resulting if you stand on the left side of $R$, the other if you stand on the right. Each punctured disk in turn, represents a punctured torus obtained by identifying the paired boundary arcs.

Thus we have explicitly described a process of pinching. The description of twisting by Method $I$ is much the same. For this case we consider the Beltrami equation on $R$,

$$
f_{\bar{z}} / f_{z}=-\frac{\text { in } / 2 M}{1+(\text { in } / 2 M)} \frac{\overline{\varphi[\gamma]}}{|\varphi[\gamma]|}, \quad M=M[\gamma]
$$

where $n$ is any positive or negative integer. The solution gives us an explicit twist $T^{n}$ of order $n$.

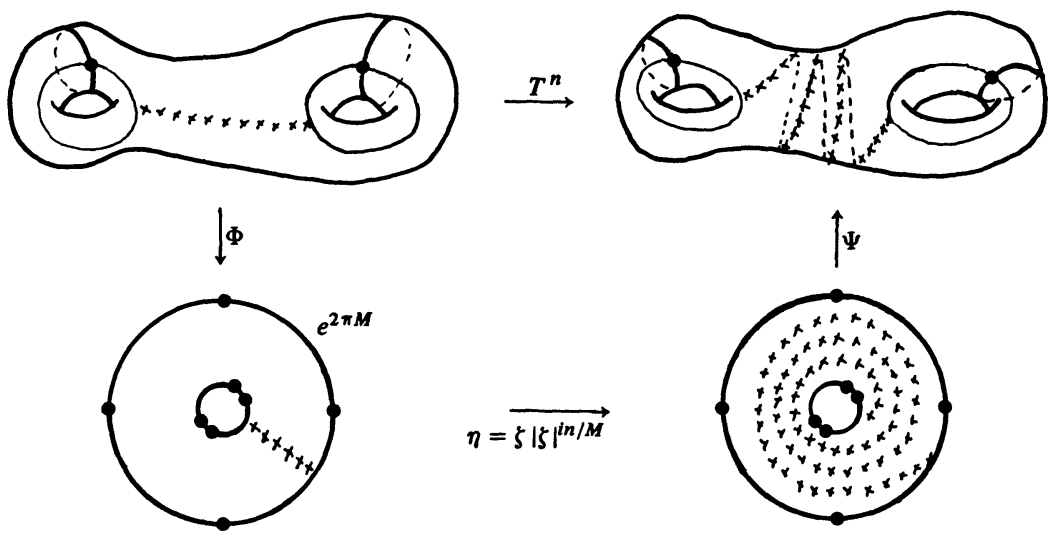


The bottom line shows the twist in the annulus, the arc between the boundary contours comes from the corresponding arc on $R$. As $n \rightarrow \infty$ there is no apparent limiting situation except that the arc becomes asymptotic to a concentric circle. One might note that the limit of the Beltrami coefficient as $n \rightarrow \infty$ is -1 , the same as when $t \rightarrow 1$ in pinching.

This description is taken from a joint paper with Masur [14].

MethoD II. The operation of pinching appears as follows in terms of simultaneous uniformization.
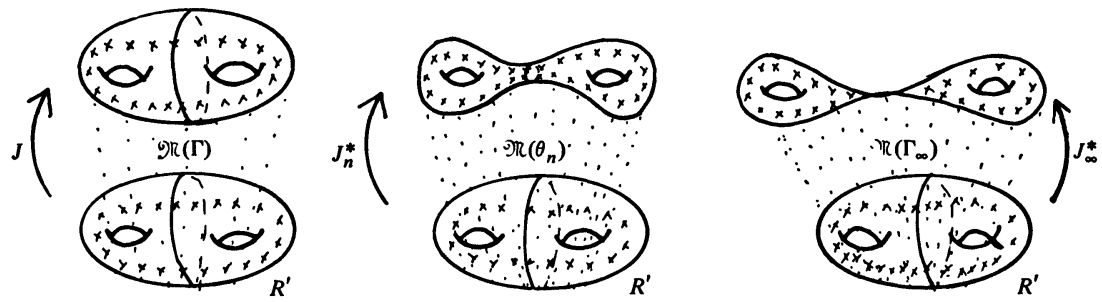

The top line consists of some of the same surfaces as in Method I. In terms of the fixed presentation $\Gamma=\left\langle\gamma_{1}, \ldots, \gamma_{2 g}\right\rangle$ of $\Gamma$, each sequence $\left\{\theta_{n}\left(\gamma_{j}\right)\right\}$ converges to a Möbius transformation $\theta_{\infty}\left(\gamma_{j}\right)$ and $\theta_{\infty}: \Gamma \rightarrow \Gamma_{\infty}=$ $\left\langle\theta_{\infty}\left(\gamma_{1}\right), \ldots, \theta_{\infty}\left(\gamma_{2 g}\right)\right\rangle$ is an isomorphism onto a kleinian group. The manifolds $\mathscr{T}\left(\theta_{n}(\Gamma)\right)$ converge to $\mathscr{R}\left(\Gamma_{\infty}\right)$ and Int $\mathscr{N}\left(\Gamma_{\infty}\right) \cong R \times(0,1)$. The reflections $J_{n}^{*}$ converge to a degenerate map $J_{\infty}^{*}$ sending $\gamma$ to a point, that is the pair of punctures, but still with the property that any simple curve $\delta \subset R^{\prime}$ and the possibly degenerate $J_{\infty}^{*}(\delta)$ bound a cylinder in Int $\mathscr{N}\left(\Gamma_{\infty}\right)$.

The operation of twisting appears in terms of simultaneous uniformization like this:
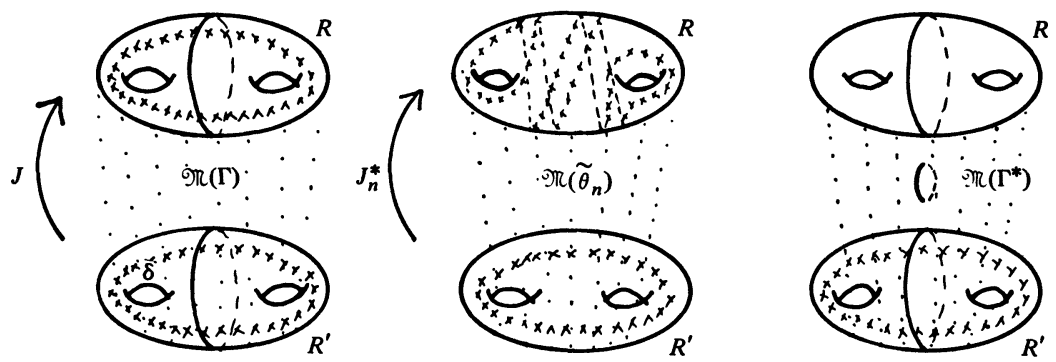

On the top line, all the surfaces are the same, namely $R$; it is only in the relation to the bottom $R^{\prime}$ that the situation is changing. If $\delta \subset R^{\prime}$ denotes the simple curve crossing $\gamma$ as indicated, $J_{n}^{*}(\delta)$ winds around $J(\gamma)$ more and more, yet bounds a cylinder in $\pi\left(\tilde{\theta}_{n}\right)$ with $\delta$. At the last step, the internal twisting in $\Re\left(\tilde{\theta}_{n}\right)$ becomes so severe that a "singular locus" must develop. The 3-manifolds $\left\{\Re\left(\tilde{\theta}_{n}\right)\right\}$ converge to $\Re\left(\Gamma^{*}\right)$ with the following properties.

(i) $\partial \mathscr{R}\left(\Gamma^{*}\right)$ has two components, one conformally equivalent to $R$, the other to $R^{\prime}$,

(ii) $\mathscr{R}\left(\Gamma^{*}\right)$ is homeomorphic to the result of removing from $\Re(\Gamma)$ a circle parallel to $\gamma$ and $J(\gamma)$. Also, $\mathscr{R}\left(\Gamma^{*}\right)$ is homeomorphic to the result of doubling $\Re\left(\Gamma_{\infty}\right)$ across the two once-punctured tori constituting its top boundary components. 
(iii) $\Gamma^{*}=\left\langle\Gamma_{\infty}, t\right\rangle$ where $t$ is a parabolic transformation. Furthermore $\lim \tilde{\theta}_{n}\left(\gamma_{j}\right)=\theta_{\infty}\left(\gamma_{j}\right), 1<j<2 g$.

We have presented two principal methods for comparing two surfaces. Method I, the generalized affine stretch, has deep connections with the geometry of curves and homeomorphisms of surfaces. Method II, placing two surfaces to be parallel boundary components of a hyperbolic 3-manifold, has ever broader ramifications. For quasifuchsian space is but the simplest of the spaces of kleinian groups-that is, discrete groups of Möbius transformations. The corresponding product manifolds are the simplest class of hyperbolic 3-manifolds.

These methods provide the basis for understanding the geometry of Teichmüller space. With this said, it is time to formally present Teichmüller space and to indicate briefly how this is so. We will stick to the case of a compact surface $R$ of genus $g$ without boundary. General references are [2] and [5].

The geometric definition of Teichmüller space $T_{g}$ is

$$
\begin{aligned}
T_{g}=\{(S, f) \mid f: R \rightarrow S \text { is an orientation preserving homeomorphism, } \\
\text { subject to the equivalence }\left(S_{1}, f_{1}\right) \equiv(S, f) \text { if and } \\
\text { only if } \left.f_{1} f^{-1}: S \rightarrow S_{1} \text { is homotopic to a conformal map }\right\} .
\end{aligned}
$$

In particular $(R$, id $)$ is treated as the origin and $(R, f) \equiv(R$, id $)$ if and only if $\boldsymbol{f}$ is homotopic to a conformal automorphism of $\boldsymbol{R}$.

By Teichmüller's theorem we may assume that each $f$ is a Teichmüller map, that is a solution of the equation $f_{\bar{z}} / f_{z}=k \bar{\varphi} /|\varphi|, 0<k<1$, for some quadratic differential $\varphi$. The distance between $(R$, id $)$ and $(S, f)$ is then defined to be $(\log K) / 2$ where $K=(1+k) /(1-k)$. Analogously, distance is defined between two general points thereby obtaining a metric, called the Teichmüller metric, on $T_{g}$. The geodesic ray emanating from $(R$, id) and passing through $(S, f)$ consists of all pairs $\left(S_{1}, f_{1}\right)$ corresponding to the same $\varphi$ as $k$ varies, $0<k<1$.

An equivalent definition of $T_{g}$ is

$$
\begin{array}{r}
T_{g}=\{\mathscr{R}(\theta): \theta(\Gamma) \text { is quasifuchsian and there is a homeomorphism } \\
\left.g: \mathfrak{R}(\Gamma) \rightarrow \mathscr{N}(\theta) \text { which is conformal on } R^{\prime} \subset \partial \mathscr{R}(\Gamma)\right\} .
\end{array}
$$

In this definition $\mathscr{R}\left(\theta_{1}\right) \equiv \mathscr{R}(\theta)$ if and only if for some Möbius transformation $T, \theta_{1}=T \theta T^{-1}$. Its correspondence to the original definition is through the relation

$$
(S, f) \leftrightarrow\left(R^{\prime}, S, f \circ J\right)
$$

with simultaneous uniformization applied to the triple to obtain $\boldsymbol{\theta}$.

$T_{g}$ is a complex analytic manifold of dimension $3 g-3$. This was first completely established by Ahlfors [1] using periods and zeros of abelian differentials. Later (see [2], [5]), Bers showed that by using the quasifuchsian groups $\{\theta(\Gamma)\}$, the complex structure could be obtained much more easily, although rather indirectly. Recently in the joint work with Earle cited previously, we show that a simple and natural geometric procedure intrinsic in the Riemann surfaces also gives rise to the complex structure. 
The group $\mathrm{Aut}^{+} R / \sim$ of orientation preserving homeomorphisms $h$ of $R$ onto itself, two being equivalent if homotopic, acts on $T_{g}$ as $(S, f) \rightarrow(S, f h)$. This is a biholomorphic automorphism in terms of the complex structure and an isometry in the Teichmüller metric. In particular the Dehn twist $T$ about $\gamma$ on $R$ so acts. This group acting in $T_{g}$ is called the Teichmüller modular group $\Gamma_{g}$. For $g=1$ it essentially reduces to the classical modular group.

While $T_{g}$ is topologically equivalent to a ball in $\mathbf{C}^{3 g^{-3}}$, its metric and complex analytic structure are quite different from that which would occur if it were also metrically or biholomorphically equivalent. Indeed, a celebrated theorem of Royden [19] asserts that the only biholomorphic automorphisms of $T_{g}$ are those of $\Gamma_{g}$, and Earle and $\mathrm{Kra}[6]$ adapted his result to show these are the only isometries as well.

Our discussion of pinching and twisting can now be summarized in the following diagram of the Teichmüller space $T_{2}$ with origin $O \equiv(R, \mathrm{id})$.

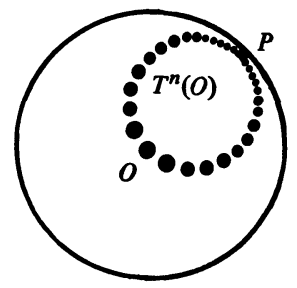

There is a boundary point or "cusp" $P$ consisting of two once-punctured tori, the point at infinity of the geodesic ray from $O$ constructed with the help of the Jenkins-Strebel differential. The cusp $P$ can be described equally well as the isomorphism $\theta_{\infty}: \Gamma \rightarrow \Gamma_{\infty}$ onto the kleinian group $\Gamma_{\infty}$. Here $\Gamma_{\infty}=$ $\left\langle\theta_{\infty}\left(\gamma_{1}\right), \ldots, \theta_{\infty}\left(\gamma_{4}\right)\right\rangle$ where $\theta_{\infty}\left(\gamma_{j}\right)=\lim \theta_{t}\left(\gamma_{j}\right)$ and the isomorphism $\theta_{t}$ corresponds to a point on the geodesic ray. Actually, $P$ lies in the "boundary space" consisting of all deformations of the two once-punctured tori.

The orbit $T^{n}(O),-\infty<n<\infty$, under the element $T \in \Gamma_{g}$ arising from the Dehn twist about $\gamma \subset R$, approaches $P$ "tangentially". This approach can be described in either of two ways. The Teichmüller disk determined by $O$ and $P$ is the image in $T_{2}$ of the unit disk $\Delta=\{\zeta \in \mathrm{C}:|\zeta|<1\}$ under the injection $\zeta \rightarrow\left(S_{\zeta}, f_{\zeta}\right)$ where $f_{\zeta}$ is a solution of $w_{\bar{z}} / w_{z}=\zeta \varphi[\gamma] /|\varphi[\gamma]|$. It is totally geodesic in $T_{2}$ and the point $\zeta=-1$ corresponds to $P$. The twist $T$ maps it onto itself and the pull-back of $T$ to $\Delta$ is a parabolic transformation with fixed point -1 [14].

Alternatively, the orbit $T^{n}(O)$ consists of a sequence of kleinian groups $\tilde{\theta}_{n}(\Gamma)$. The 3-manifolds $\Re\left(\tilde{\theta}_{n}\right)$ do not converge to the product manifold $\Re\left(\Gamma_{\infty}\right)$ but rather to $\Re\left(\Gamma^{*}\right)$. This is because the generators $\left\{\tilde{\theta}_{n}\left(\gamma_{j}\right)\right\}$ of $\tilde{\theta}_{n}(\Gamma)$, although converging to the generators $\left\{\theta_{\infty}\left(\gamma_{j}\right)\right\}$ of $\Gamma_{\infty}$, do so "tangentially" in terms of the coefficients. This phenomenon was described by Jorgensen [11] and we must refer there for details.

In conclusion, I have tried to expose the geometric underpinnings of Teichmüller space theory. Inevitably and perhaps surprisingly, we are led to 3-dimensional hyperbolic manifolds. On a much grander scale, the recent results of Thurston show that the study of such manifolds to a large extent 
coincides with the study of general 3-dimensional manifolds. Yet this can all be viewed as but one aspect of the global study of analytic functions. We at Minnesota are bullish on complex analysis.

\section{REFERENCES}

1. L. V. Ahlfors, The complex analytic structure of the space of closed Riemann surfaces, Analytic Functions (R. Nevanlinna et al, eds.), Princeton Univ. Press, Princeton, N. J., 1960, pp. 45-66.

2. __ Lectures on quasiconformal mappings, Van Nostrand, Reinhold, Princeton, N. J., 1966.

3. L. Bers, On boundaries of Teichmüller spaces and kleinian groups. I, Ann. of Math. (2) 91 (1970), 570-600.

4. Q Quasiconformal mappings and Teichmüller's theorem, Analytic Functions (R. Nevanlinna et al, eds.), Princeton Univ. Press, Princeton, N. J., 1960, pp. 89-119.

5. C. Earle, Teichmüller theory, Discrete Groups and Automorphic Functions (W. Harvey, ed.), Academic Press, London, 1977, pp. 143-161.

6. C. Earle and I. Kra, On isometries between Teichmüller spaces, Duke Math. J. 41 (1974), 583-591.

7. C. Earle and A. Marden, Quasifuchsian space and geometric complex coordinates for Teichmüller and moduli space (to appear).

8. F. W. Gehring, Rings and quasiconformal mappings in space, Trans. Amer. Math. Soc. 103 (1962), 353-393.

9. J. Hubbard and H. Masur, Quadratic differentials and foliations, Acta Math. 142 (1979), 211-274.

10. J. A. Jenkins, On the existence of certain general extremal metrics, Ann. of Math. (2) 66 (1957), 440-453.

11. T. Jorgensen, On reopening of cusps (to appear).

12. S. P. Kerckhoff, The asymptotic geometry of Teichmüller space, Thesis, Princeton Univ., 1978; Topology 19 (1980), 23-41.

13. A. Marden, Geometrically finite kleinian groups and their deformation spaces, Discrete Groups and Automorphic Functions (W. Harvey, ed.), Academic Press, London, 1977, pp. 259-293.

14. A. Marden and H. Masur, A foliation of Teichmüller space by twist invariant disks, Math. Scand. 36 (1975), 211-228; 39 (1976), 232-238.

15. A. Marden and K. Strebel (to appear).

16. B. Maskit, On boundaries of Teichmüller space. II, Ann. of Math. (2) 91 (1970), 607-639.

17. H. Masur, On a class of geodesics in Teichmüller space, Ann. of Math. (2) 102 (1975), 205-221.

18. The Jenkins-Strebel differentials with one cylinder are dense, Comment. Math. Helv. 54 (1979), 179-184.

19. H. L. Royden, Automorphisms and isometries of Teichmüller space, Advances in the Theory of Riemann Surfaces (L. Ahlfors et al, eds.), Ann. of Math. Studies, no. 66, Princeton Univ. Press, Princeton, N. J., 1971, pp. 369-383.

20. K. Strebel, On quadratic differentials and extremal quasiconformal mappings, Univ. of Minnesota Lecture Notes, 1967.

21. __ Quadratic differentials, Springer-Verlag (Ergebnisse series) (to appear).

22. A. Fathi, F. Laudenbach and V. Poénaru, Travaux de Thurston sur les surfaces, Astérisque 66-67 (1979).

23. W. Thurston, The geometry and topology of 3-manifolds, Lecture Notes, Princeton Univ.

Department of Mathematics, University of Minnesota, Minneapolis, Minnesota 55455 
